\title{
Temporal regulation of gene expression in the blastoderm Drosophila embryo
}

\author{
Glenn K. Yasuda, ${ }^{1,3}$ Jayne Baker, ${ }^{2}$ and Gerold Schubiger ${ }^{2}$ \\ ${ }^{1}$ Department of Genetics, SK-50, and ${ }^{2}$ Department of Zoology, NJ-15, University of Washington, \\ Seattle, Washington 98195 USA
}

\begin{abstract}
The Drosophila embryo undergoes a developmental transition during cycle 14 when it initiates asynchronous mitotic cycles and markedly increases its rate of zygotic transcription. The nucleo-cytoplasmic ratio has been proposed to be the single factor that temporally regulates this developmental transition. We altered the ratio in the embryo and analyzed the consequences on the cell cycle program and on the transcripts of specific genes. These genes were chosen because their transcripts normally undergo changes in pattern during cycle 14. We found evidence that the nucleo-cytoplasmic ratio is read and interpreted locally to regulate the cell cycle program. Based on the response of the transcripts to changes in the ratio, we found evidence that at least two classes of temporal regulatory mechanisms control these transcripts. We therefore propose two corresponding classes of transcripts: (1) nucleo-cytoplasmic ratio dependent; and (2) nucleo-cytoplasmic ratio independent or time correlated. The temporal regulation of the ratio-independent transcripts may be dependent on developmental time. We conclude that multiple modes of temporal regulation underlie the events of the developmental transition in Drosophila embryogenesis.
\end{abstract}

[Key Words: Temporal regulation; nucleo-cytoplasmic ratio; cell cycle; maternal message degradation; zygotic transcription]

Received April 9, 1991; revised version accepted August 13, 1991.

Development is a four-dimensional process. Although a great deal is known about the spatial regulation of development, much less is known about the temporal regulation of early developmental events. The morphogenetic movements and molecular changes must be correlated in time and in space to produce the coherent developmental process. In several invertebrate and vertebrate organisms, mitotic synchrony is lost and the zygotic genome is transcriptionally activated in the late blastula stages just prior to gastrulation. This transition is therefore a crucial time for the coordination of morphogenetic and molecular events. The morphogenetic events of this transition have been described in several organisms. However, the temporal regulation of the concerted morphogenetic and molecular transitions is not understood.

In Drosophila melanogaster early development, this developmental transition occurs during cycle 14 . The cell cycle program of the early embryo consists of a series of rapid, synchronous mitotic divisions as a syncytium (Zalokar and Erk 1976; Foe and Alberts 1983). The initial nine cycles, which are $\sim 10 \mathrm{~min}$ long, take place in the center of the embryo. After migration to the embryo surface, the nuclei undergo four additional blastoderm divisions prior to cellularization. The length of interphase in these cycles is progressively longer. During cycle 14,

\footnotetext{
${ }^{3}$ Corresponding author.
}

which is at least $60 \mathrm{~min}$ long, there is a major transition in the embryo as the nuclei are cellularized, the mitotic synchrony is lost, and gastrulation movements are initiated. These morphological changes are coincident with the segmental determination of the embryonic cells (Simcox and Sang 1983) and the transition from maternal to zygotic control of the cell cycle program (Edgar et al. 1986a; Edgar and O'Farrell 1989).

The transition from the synchronous, maternally controlled cycles to the asynchronous, zygotically controlled cycles is thought to involve the imposition of additional products that regulate a basic oscillator (for review, see Murray and Kirschner 1989). In a number of organisms including Drosophila, there is evidence that the timing of this transition is regulated by the nucleocytoplasmic ratio /Chulitskaia 1970; Signoret and Lefresne 1971; Kobayakawa and Kubota 1981; Newport and Kirschner 1982; Edgar et al. 1986). In Drosophila, the ratio apparently also regulates the basic oscillator to produce the progressively longer cycle lengths observed in cycles 10-13. The nucleo-cytoplasmic ratio therefore has a major effect on the cell cycle program of the early embryo.

At the molecular level, a transition also occurs in cycle 14 when transcription of all classes of genes is massively increased and the genome reaches its greatest transcriptional activity during embryogenesis (Lamb and Laird 1976; McKnight and Miller 1976; Anderson and 
Lengyel 1979; Edgar and Schubiger 1986). Information on transcriptional activation has come from studies of pulse-labeled RNA from precisely staged embryos and from drug studies that examine the effects of alterations in the cell cycle program on the levels of zygotic transcription. These results led to the proposal that at the time of nuclear migration all genes become competent for transcription; the actual transcription levels are regulated by the length of interphase, which is in turn, regulated by the nucleo-cytoplasmic ratio (Edgar and Schubiger 1986). A similar mechanism has been proposed to regulate transcriptional activation at the mid-blastula transition in Xenopus (Kimelman et al. 1987). The nucleocytoplasmic ratio is therefore also proposed to have a major effect on the temporal regulation of changes in transcript pattern and accumulation.

In addition to this general mechanism for temporal regulation by interphase time, there is some evidence for another level of temporal programming of transcriptional activation in Drosophila. Edgar and Schubiger (1986) noted that although transcripts from numerous genes are first detected in cycle 11 or 12 , some genes reach maximal activation prior to others. Cloning and characterization of the gap class of zygotic segmentation genes has revealed that these genes are transcribed in cycle 12 and are strongly expressed in cycle 13 (Knipple et al. 1985; Tautz et al. 1987; Rothe et al. 1989). It is not known whether this temporal program of transcription is also sensitive to changes in the cell cycle program.

In principle, it is possible that the loss of mitotic synchrony and the activation of the zygotic genome characteristic of the transition in cycle 14 are both regulated by the nucleo-cytoplasmic ratio. To investigate this possibility, we examined the effects of an altered nucleocytoplasmic ratio on transcripts that normally undergo changes in transcript pattern in cycle 14. UV irradiation and DNA injection were used to reproducibly induce the loss of nuclei and generate embryos with a decreased nuclear density. Our observations extend the hypothesis that the cell cycle program is controlled by the nucleocytoplasmic ratio. However, we find that the transcripts from some genes were not effected by the decreased nucleocytoplasmic ratio. These genes were apparently regulated by developmental time. Thus, we propose that the events of the developmental transition from maternalto-zygotic information during cycle 14 must be regulated by multiple independent modes of temporal regulation.

\section{Results \\ Embryos survive the loss of nuclei induced by UV irradiation and DNA injection}

Togashi and Okada (1984) demonstrated that polar UV irradiation prior to migration causes mitotic arrest of the underlying nuclei. These nuclei remain arrested with a metaphase/anaphase-like morphology. When one pole of the embryo was irradiated at cycle 6 , the effect was obvious following nuclear migration to the periphery at cycle 10. The embryo in Figure la was irradiated at the
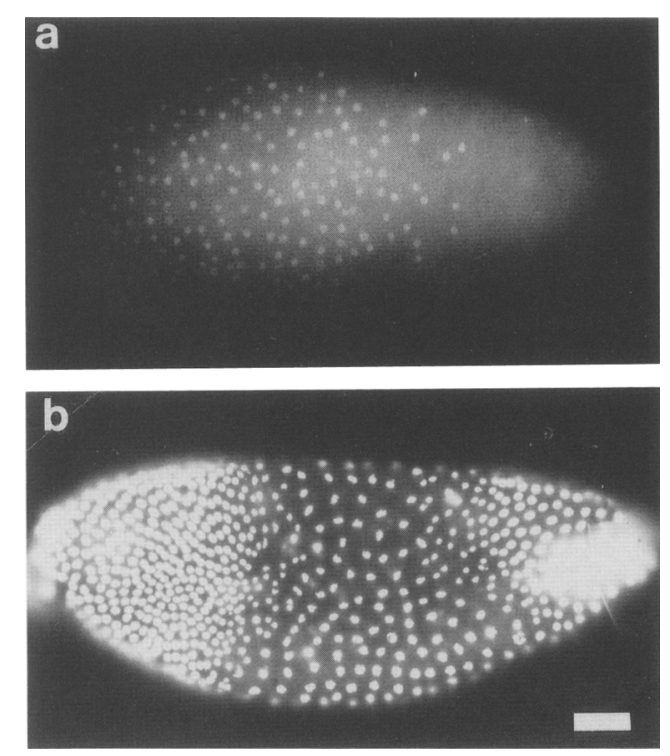

Figure 1. Nuclear loss is induced by UV irradiation and DNA injection at cycle 6. Experimental embryos were stained with DAPI. $(a)$ The UV-irradiated embryo is in interphase of cycle 11. One full cycle after nuclear migration at cycle 10, the nuclei are present only in the posterior half of the embryo. $(b)$ The DNAinjected embryo is in interphase of cycle 14. Clearly, there are regions of the embryo with a decreased nuclear density. Anterior is to the right. Bar, $50 \mu \mathrm{m}$.

anterior pole at cycle 6 and, in cycle 11, only has nuclei at the periphery of the unirradiated posterior half. We found that embryos irradiated at the anterior pole were able to compensate for this nuclear loss prior to cellularization. Although there were often regional differences in nuclear density across the embryo, the compensation process resulted in embryos with an overall nuclear density at cellularization that was greater than that of control cycle 13 embryos but less than that of control cycle 14 embryos. The irradiated embryos developed cuticle $92 \%$ of the time and hatched $41 \%$ of the time $(n=91)$ relative to controls $(n=70)$. Of the hatching larvae, $42 \%$ $(n=30)$ survived to adulthood relative to controls (n=50).

In contrast to the UV-irradiated embryos, which only had nuclei in the unirradiated end, the DNA-injected embryos did not have a significantly lowered nuclear density at cycle 10 . The mitotic cycles up to cycle 14 were rapid as in the control embryos. The mitotic waves, however, did not result in a doubling in the number of nuclei. The loss of nuclei was therefore progressive, and the difference in nuclear density between DNA-injected and control embryos increased with each division (data not shown). The DNA-injected embryo in Figure $1 \mathrm{~b}$ has large regions with a lowered nuclear density. In these embryos, the injected DNA becomes vesiculated and surrounded by a nuclear membrane (Yasuda et al. 1991). We believe that the titration of materials involved in membrane formation resulted in the observed loss of dividing host nuclei. The DNA-injected embryos devel- 
oped cuticle $57 \%$ of the time $(n=36)$ relative to bufferinjected controls $(n=70)$.

\section{$U V$-irradiated and DNA-injected embryos undergo additional mitotic divisions prior to cellularization to compensate for the loss of nuclei}

UV-irradiated and DNA-injected embryos were observed by timelapse video microscopy and direct observation of living embryos on a compound microscope. We found that the embryos were able to compensate for the nuclear loss by altering their cell cycle program prior to cellularization. Careful timing of the mitotic cycles is extremely informative because there is a progressive increase in cycle length of the blastoderm divisions $(\mathrm{Za}$ lokar and Erk 1976; Foe and Alberts 1983). This cycle length progression provides a reliable metric for detecting changes in the development of the experimental embryos.

An example of the steps involved in the compensation process is illustrated in Figure 2. Following nuclear migration at the normal cycle 10 time, only the unirradiated posterior half of the embryo had nuclei at the surface (Fig. 2a). More than $90 \%$ of the irradiated embryos had this initial phenotype $(n=160)$. During the subsequent five mitotic cycles $\mid$ Fig. $2 \mathrm{~b}$ represents cycle 13; Fig. 2c represents cycle 15), the nuclei spread across

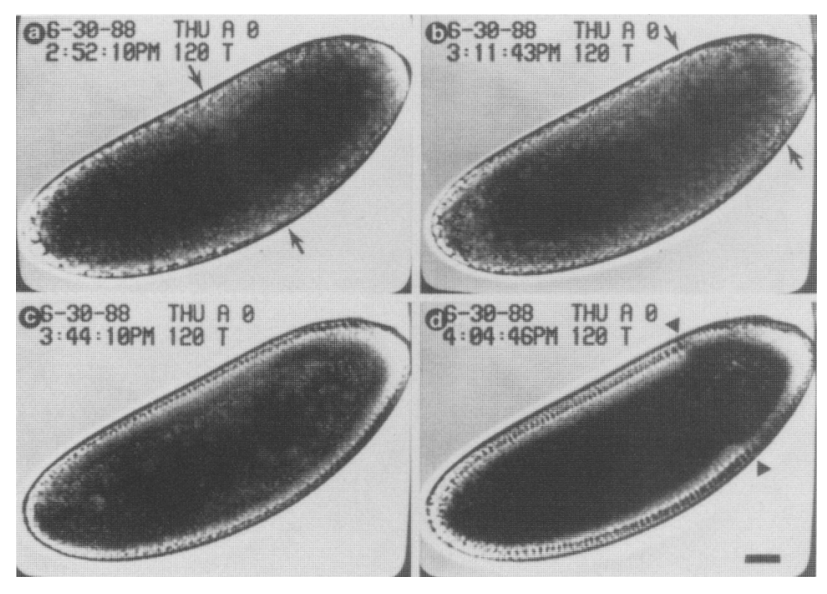

Figure 2. Compensation for the loss of nuclei induced by UV irradiation requires the initiation of additional mitotic cycles prior to gastrulation. The development of UV-irradiated embryos was monitored by time-lapse video microscopy. $(a)$ After migration, there are nuclei at the surface of only the unirradiated posterior half of the embryo. In $a$ and $b$, the anterior-most nuclei visible is denoted with the arrows. $\{b \mid$ In cycle 13 , nuclei have shifted forward during divisions so that they are now extended to $\sim 75 \%$ of the embryo length. (c) In cycle 15 , there are nuclei along the entire length of the embryo, but the nuclear density is lower at the anterior pole. (d) The embryo underwent a partial cycle 16 , which only involved the nuclei in the anterior fifth of the embryo, prior to gastrulation. The boundary of the region undergoing the extra local division is marked by arrowheads. The anterior fifth of the embryo initiated gastrulation movements significantly later than the remainder of the embryo. The anterior end of the embryo is at the upper right. Bar, $50 \mu \mathrm{m}$. the entire length of the embryo but the nuclear density was still low at the irradiated anterior pole. The mitotic waves up to this point were rapid, initiated at the anterior pole of the embryo, and included all of the nuclei. During these mitotic waves, the nuclei streamed across the embryo and filled in the empty anterior cortex. This was in contrast to the symmetrical mitotic waves initiated from both poles that were observed in control embryos. The last mitotic wave was slow and extended about one-fifth of the way across the embryo (Fig. 2d). This particular embryo, which had undergone an additional complete mitotic wave and an additional local mitotic wave, developed into a normally segmented, firstinstar larva.

While the compensation process of each embryo varied in detail, the pattern of divisions fell into three basic classes (Fig. 3a), (1) Anterior 15: embryos in which only a local region at the anterior end underwent an additional (fifteenth) round of division $144 \%$ of the irradiated embryos scored by video or direct observation; $n=30$ ). The cell cycle program prior to cycle 14 did not differ from that of the control embryos, but cycle 14 was subdivided by the additional division in the anterior portion of the embryo. (2) Complete 15: embryos in which the entire population of nuclei underwent an additional mitotic division (26\% of the embryos). An additional cycle, similar in length to cycle 10, was inserted into an apparently normal cell cycle program. (3) Anterior 16: embryos that underwent both an additional complete mitotic wake, followed by an additional local mitotic wave ( $30 \%$ of the embryos). In these embryos the cell cycle program was altered so that cycle 14 was earlier than cycle 14 in the control embryos and cycle 15 was earlier than cycle 15 in the complete 15 class of irradiated embryos. All three classes of compensation have an altered cell cycle program during the time interval that covers cycle 14 in the unirradiated control embryos.

The pattern of compensating divisions in the DNAinjected embryos fell into two classes (Fig. 3b). (1) Partial 15: embryos that underwent an additional mitoses of a fraction of the embryonic nuclei. (2) Complete 15: embryos that underwent an additional complete mitotic wave. In contrast to the observations on UV-irradiated embryos, the two classes of compensating DNA-injected embryos do not differ significantly from each other or from the control embryos in cell cycle program prior to cycle 14. The extra divisions subdivide a slightly extended cycle 14. This is consistent with the progressive loss of nuclei during the mitotic cycles.

In summary, the developing embryo normally undergoes a series of stereotypical, blastoderm divisions and then cellularizes and gastrulates. If the normal doubling in nuclear number was disrupted in the early embryo, the embryo changed its cell cycle program by initiating additional mitotic divisions prior to cellularization. These divisions could have involved all of the nuclei or a subset of the nuclei and resulted in a nuclear density at cellularization that was greater than that of a cycle 13 control embryo. The extra divisions did not block the continued development of the embryos. 


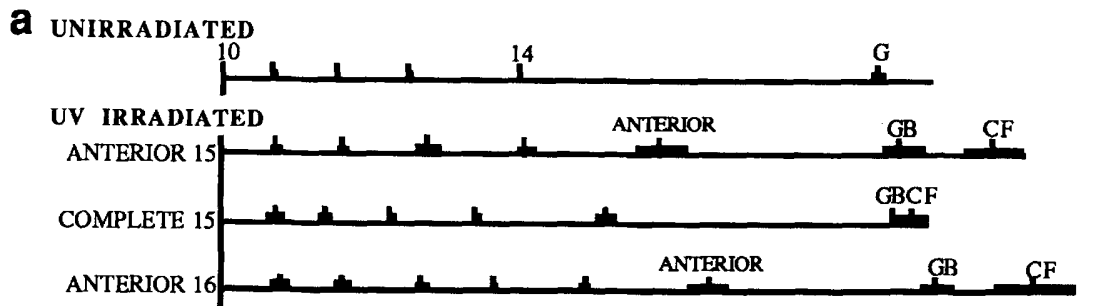

b.

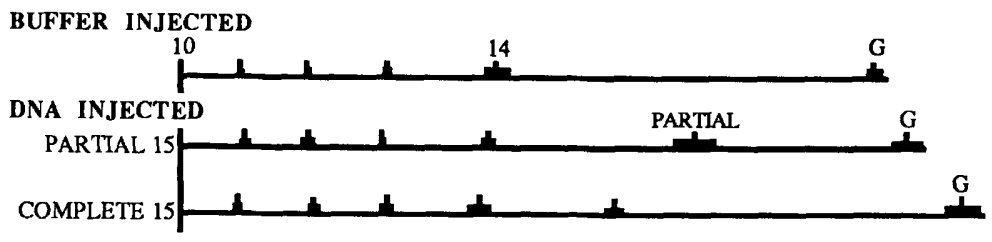

Figure 3. Summary of the cell cycle program in the UV-irradiated and DNA-injected embryos analyzed by time-lapse video microscopy. The mean and S.M. in cell cycle length are denoted by the vertical and horizontal bars, respectively. $(a)$ On the basis of the observed cell cycle program of the embryos, the UV-irradiated embryos have been divided into three classes (described in the text): (1) anterior $15(n=3) ;(2)$ complete $15(n=4)$; and (3) anterior $16(n=3)$. Anterior indicates the anterior-limited extra division. Parallel observations from unirradiated embryos are controls used for comparison of cycle length progression $(n=8)$. (b) On the basis of the observed cell cycle program of the embryos, the DNAinjected embryos have been divided into two classes (described in the text): (1) partial $15(n=7)$; and (2) complete $15(n=5)$. Partial indicates the extra division in a subpopulation of the nuclei. Parallel observations from injection buffer-injected embryos are controls used for comparison of cycle length progression $(n=11)$. Numbers and abbreviations: $(10)$ cycle $10 ;(14)$ cycle 14 ; (G) gastrulation; (GB) germ-band movement; (CF) cephalic furrow formation.

\begin{abstract}
A delay in the maternal-to-zygotic transition of the mitotic control gene string was correlated with a decrease in nuclear density and a change in the cell cycle program
\end{abstract}

Because UV irradiation and DNA injection resulted in changes in the cell cycle program, we examined the effects of these manipulations on the transcripts of the mitotic control gene string (stg). The stg transcript pattern undergoes a dramatic maternal-to-zygotic transition during cycle 14 (Edgar and $O^{\prime}$ Farrell 1989). The transcript is supplied maternally at high levels and is maintained stably through the beginning of cycle 14 . It is rapidly degraded early in cycle 14. Shortly before the initiation of gastrulation, zygotic transcription of stg is initiated in a spatially and temporally specific pattern. This pattern accurately predicts the mitotic domains that will be activated $\sim 30$ min after each stg domain can be detected (Edgar and O'Farrell 1989; Foe 1989). This observation and results from experiments in which stg was ectopically transcribed have led to the proposal that the $s t g$ product is a dose-dependent inducer of mitosis after cycle 14 (Edgar and O'Farrell 1989, 1990).

Embryos were UV irradiated or DNA injected and fixed in parallel with the unirradiated or buffer-injected controls, respectively. stg transcript was then visualized by in situ hybridization. The embryos were scored as belonging to one of four classes (Fig. 4a): (1) Maternal (M)-high level uniform transcript; (2) degraded (D)-no or low levels of transcript; (3) early zygotic (EZ)-a ventral stripe of transcript (the first detectable zygotic transcription); and (4) zygotic (Z)-additional zygotic pattern elements. Early in cycle 14 in the control embryos [170 $\pm 5 \mathrm{~min}$ after egg laying (AEL)], more than one-third $(36 \%)$ of the control embryos had progressed to the degraded class. Only $6 \%$ of the UV-irradiated embryos had degraded stg maternal message. In the middle of cycle 14 in the controls $(200 \pm 5 \mathrm{~min}$ AEL), almost half $(49 \%)$ of the control embryos had zygotic stg transcription. Only
$2 \%$ of the irradiated embryos fixed at the same time had stg transcription. Late in cycle 14 in the controls $(230 \pm 5 \mathrm{~min}$ AEL), $75 \%$ of the control embryos and only $18 \%$ of the irradiated embryos had progressed to the zygotic class. The irradiated embryos were therefore significantly delayed throughout the cycle 14 time period, both in the degradation of maternal stg message and in the initiation of zygotic stg transcription. Because degradation and transcription initiation were apparently delayed to the same extent, the simplest interpretation is that the onset of the stg transition was delayed but that the programmed changes from maternal transcript to patterned zygotic transcription occurred over a normal time span. DNA-injected embryos were delayed in the maternal-to-zygotic transition of stg expression when compared to buffer-injected controls at both the 200-min time point $(\mathrm{P}<0.01, n=53$ for controls and $n=76$ for DNA-injected embryos| and the 230-min time point $(\mathrm{P}=0.05, n=36$ for controls and $n=55$ for DNA-injected embryos). The two very different experimental manipulations both caused the loss of nuclei. Therefore, the most obvious explanation is that the decreased nuclear density and/or the resulting change in cell cycle program must somehow be read and interpreted to regulate the maternal-to-zygotic transition in stg expression.

There were also UV-irradiated and DNA-injected embryos that were mosaic in their stg expression pattern. That is, different parts of an embryo could be scored as belonging to different classes of $s t g$ expression pattern. These embryos had undergone additional local divisions and were therefore also mosaic in their mitotic pattern. The normal stg zygotic expression pattern is shown in Figure $4 \mathrm{~b}$. Following the ventral stripe expression, the first domains of stg zygotic expression are at the anterior end in the prospective head regions. These domains are evident prior to transcription in the posterior domains of expression. Figure $4 \mathrm{c}$ shows the DAPI staining of a UVirradiated embryo. This embryo apparently underwent 
Figure 4. The maternal-to-zygotic transition in transcript pattern (TXN) of the mitotic control gene stg is delayed in the UV-irradiated embryos. UV-irradiated and unirradiated controls were fixed in parallel at 170,200, and $230 \mathrm{~min} \mathrm{AEL}$, and the $s t g$ expression pattern was visualized by in situ hybridization. The embryos were then scored as belonging to one of four classes of stg expression pattern. The details of the scoring classes are described in the text. (a) Summary of the observations made on irradiated (hatched bars) and unirradiated (solid bars) embryos at the three time points. Sample sizes for 170-, 200-, and 230min time points were 33,59 , and 60 , respectively, for irradiated embryos and 52,70, and 140 , respectively, for unirradiated embryos. The stg expression pattern in the irradiated embryos is delayed significantly at the 170 $\min (\mathrm{P}<0.01)$, the $200-\mathrm{min}(\mathrm{P}<0.001)$, and the 230-min $(P<0.001)$ time points when compared to the pattern in the control embryos. In situ hybridization of the unirradiated embryo in $b$ shows the normal zygotic stg expression pattern. In $b-d$, the embryos are aligned with ventral in front and anterior to the right. The ventral stripe of zygotic transcript is denoted with the arrowhead. The DAPI image of the irradiated embryo in $c$ shows the nuclear morphology differences observed as a result of the additional local mitotic division in the anterior third of the embryo. The inset shows the differences in morphology at the boundary of the region at a magnification $6 \times$ higher. In situ hybridization of this irradiated embryo in $d$ shows the normal zygotic stg expression pattern, ventral stripe, and some posterior domains in the region of the embryo that did not undergo the extr. division. The ventral stripe of zygotic transcript is denoted by the arrowhead. Bar, $50 \mu \mathrm{m}$.

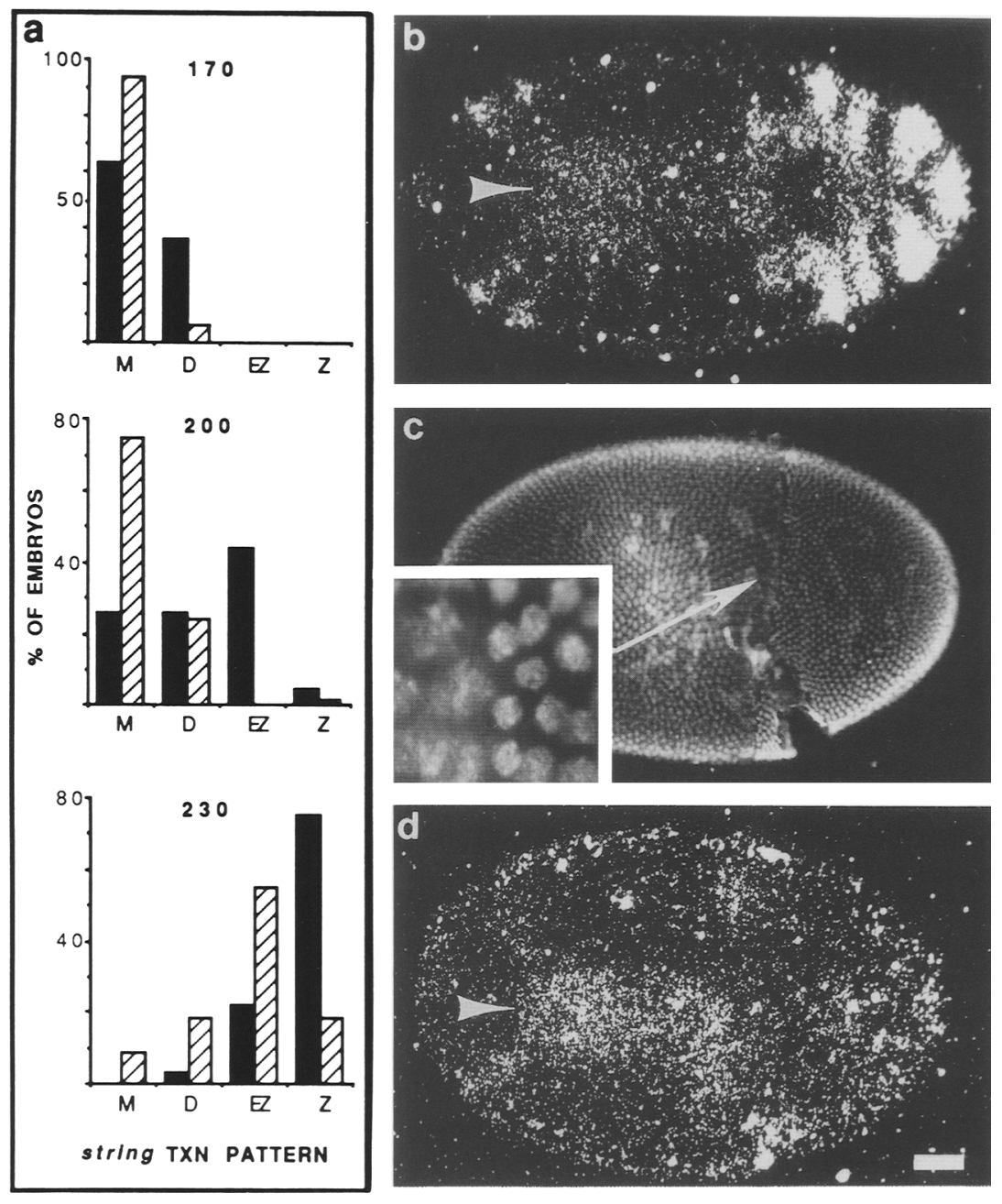

ra division and a degraded expression pattern in the region that underwent the extra

an anterior 15 type of compensation for nuclear loss. The inset in Figure 4c shows the clear difference in nuclear morphology at the boundary of the region that had undergone the additional mitosis. Figure $4 \mathrm{~d}$ shows the $s t g$ expression of this embryo. The ventral stripe and posterior domains of expression are present, but there is no evidence of the anterior domains of expression. The anterior portion of the embryo, which had undergone an additional mitotic wave, has degraded stg RNA while the remainder of the embryo has a zygotic stg expression pattern. The decreased nuclear density and/or the altered cell cycle program may therefore be read and interpreted locally in the embryo to regulate the maternal-to-zygotic transition of stg expression.

\section{A delay in the maternal-to-zygotic transition of the dorsal-ventral polarity gene Toll was correlated with a decrease in nuclear density and a change in the cell cycle program}

Maternal expression of the Toll (Tl) gene is required for correct dorsal-ventral patterning in the embryo (Ander- son et al. 1985). In addition, zygotic transcription is required for larval viability (Gerttula et al. 1988). Transcript from the $T l$ gene undergoes a maternal-to-zygotic transition during cycle 14, (Gerttula et al. 1988; Hashimoto et al. 1988). However, the transition from uniform maternal message to patterned zygotic message takes place at the end of cycle 14 and there is a significant interval when both maternal and zygotic message are present.

The maternal-to-zygotic transition of the $T l$ transcript in UV-irradiated embryos was examined by the same method described above for stg. After in situ hybridization to visualize the $T l$ transcript, the embryos were scored for their transcript pattern into three classes (Fig. 5a): (1) Uniform (U)-uniform maternal transcript; (2) basal (B) - degradation of maternal message results in a distinct concentration of the remaining transcript at the basal side of the blastoderm nuclei; and (3) zygotic $(\mathrm{Z})$ zygotic transcription of $\mathrm{Tl}$ at the anterior and posterior poles of the embryo. At the middle cycle 14 time point ( $200 \pm 5 \mathrm{~min}$ AEL), $71 \%$ of the irradiated embryos and only $44 \%$ of the control embryos had uniform maternal 
transcript. In addition, $10 \%$ of the control embryos and none of the irradiated embryos had zygotic $T 1$ transcription. At the late cycle 14/early gastrulation time point (260 $\pm 5 \mathrm{~min}$ AEL), $61 \%$ of the control embryos and only $28 \%$ of the irradiated embryos had zygotic $T 1$ transcription. The irradiated embryos were therefore significantly delayed both in the degradation of maternal $T I$ transcript and the initiation of zygotic $T 1$ transcription.

As was observed with $s t g$, the dependence of the $T I$ maternal-to-zygotic transition on nuclear density and cell cycle program could be observed in embryos mosaic for their cell cycle program. Figure $5 b$ shows the DAPI nuclear staining of an embryo that underwent an anterior 15 type of compensation with a mitotic wave, which initiated at the anterior pole and spread approximately two-thirds of the way across the embryo. The $T I$ in situ staining is shown in Figure 5c. The anterior two-thirds of the embryo has moderate levels of maternal message concentrated at the basal side of the blastoderm nuclei. The posterior one-third of the embryo has low maternal message and zygotic $T 1$ transcript at the posterior pole. There is an intermediate level of staining at the boundary, which may indicate that either $T l$ transcript or the proteins that degrade $T l$ transcript diffuse locally in the embryo.

A delay in the degradation of the bicoid maternal message was correlated with a decrease in nuclear density and a change in the cell cycle program

To ask whether the delay in degradation of maternal stg and $T 1$ messages was indicative of a delay in degradation of other maternal messages, we examined the transcripts of the maternal pattern control gene bicoid (bcd). The $b c d$ transcript is maternally loaded into the anterior end of the oocyte (Frigerio et al. 1986; Berleth et al. 1988) and is stably maintained through cycle 14 . The bcd message is then rapidly degraded at the beginning of cycle 14 . This degradation of $b c d$ message is concurrent with degradation of the stg message.

UV-irradiated and control embryos were fixed in parallel and then hybridized in situ to reveal the pattern of bcd transcript. The embryos were scored into two classes (Fig. 6): (1) Plus (+) indicates maternal transcript at the anterior pole; and (2) minus $(-)$ indicates no transcript. As both samples at the early cycle 14 time point were predominantly in the plus class, breakdown of $b c d$ maternal message was apparently initiated later than breakdown of stg message. There was no significant difference between the two samples of embryos at this time point $(\mathrm{P}=0.08)$. However, $5 \%$ of the unirradiated embryos and none of the irradiated embryos had degraded their bcd transcript. At the other two time points, there was a significant difference between the two samples. At the middle cycle 14 time point ( $200 \pm 5 \mathrm{~min}$ AEL), $83 \%$ of the irradiated embryos and only $40 \%$ of the control embryos had bcd maternal transcript. At the late cycle 14 time point $(230 \pm 5 \mathrm{~min}$ AEL), $79 \%$ of the control embryos and about half $(51 \%)$ of the irradiated embryos had degraded their maternal bcd transcript. Therefore, the decreased nuclear density and resulting change in the

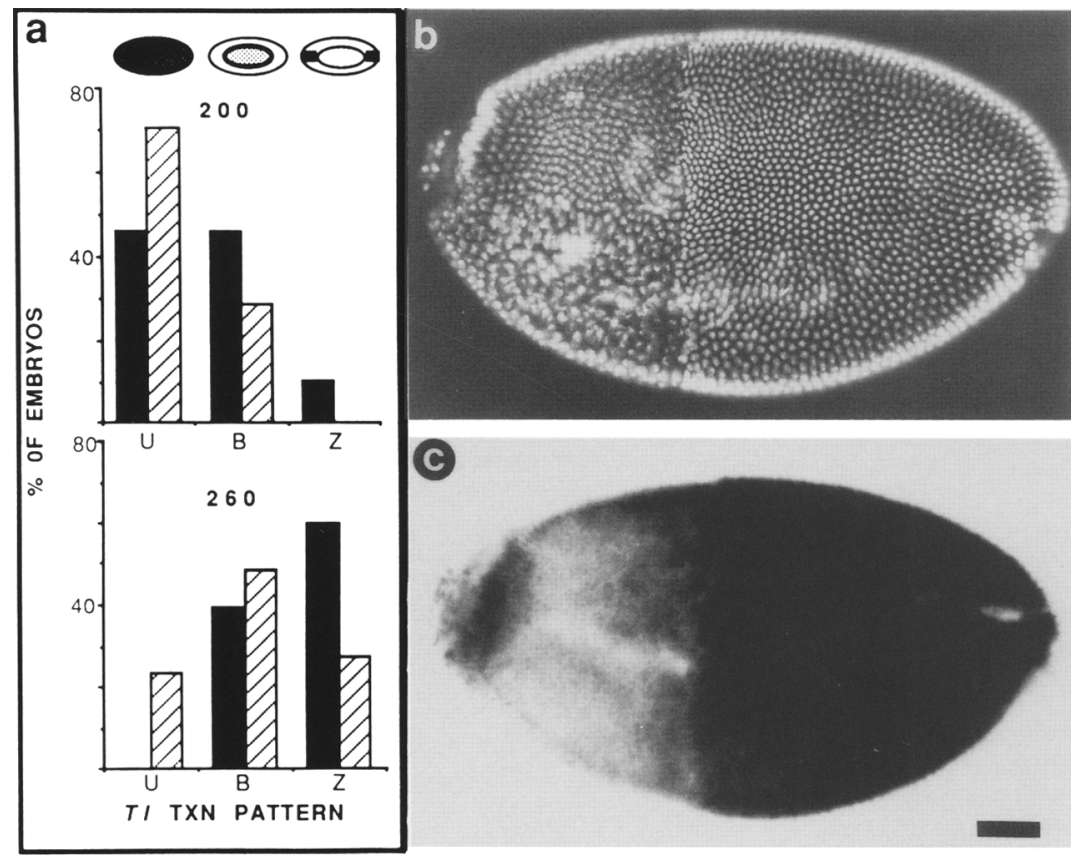

Figure 5. The maternal-to-zygotic transition in transcript pattern (TXN) of the dorsal-ventral gene $T l$ is delayed in the UV-irradiated embryos. UV-irradiated and unirradiated controls were fixed in parallel at 200 and $260 \mathrm{~min}$ AEL. The $T l$ transcript was visualized by hybridization with a nonradioactive probe, followed by detection with an antibody conjugated to alkaline phosphatase. The embryos were then scored as belonging to one of three classes. The details of the scoring classes are described in the text and are diagramed schematically in $a$. (a) Summary of the observations made on the irradiated (hatched bars) and unirradiated (solid bars) embryos at the two time points. Sample sizes for 200- and 260-min time points were 45 , and 90 , respectively, for irradiated and 31 and 38 for unirradiated embryos. The $T l$ expression pattern in the irradiated embryos is delayed significantly at the $200-\mathrm{min}(\mathrm{P}<0.05)$ and the 260-min $(P<0.001)$ time points when compared to the pattern in the control embryos. The DAPI image of the irradiated embryo in $b$ shows the nuclear morphology differences observed as a result of the additional local mitotic division in the anterior two-thirds of the embryo. In situ hybridization of this irradiated embryo in $c$ shows lower levels of maternal transcript and zygotic $T l$ expression at the posterior pole in the region of the embryo that did not undergo the extra division and higher levels of maternal transcript in the region of the embryo that underwent the extra division. Bar, $50 \mu \mathrm{m}$. 

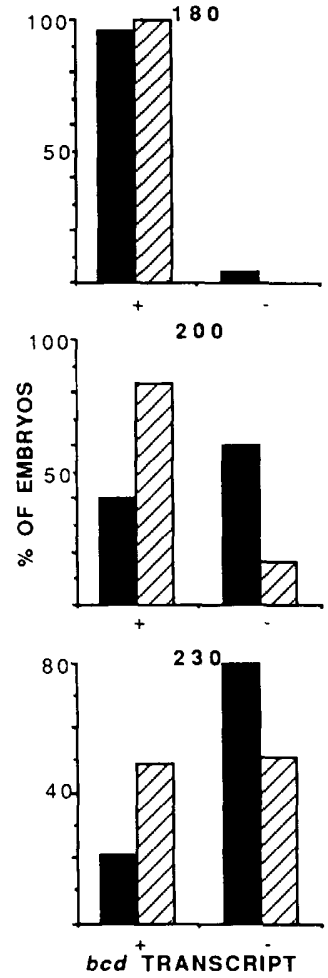

Figure 6. The degradation of $b c d$ maternal message is delayed in the UV-irradiated embryos. Irradiated and unirradiated embryos were fixed in parallel at 180-, 200-, and 230-min AEL, and $b c d$ expression was then visualized by in situ hybridization. The embryos were scored for the persistence of maternal bcd message. While there is no significant difference at the early time point $(\mathrm{P}=0.08)$, irradiated embryos (hatched bars) were significantly delayed in bcd degradation when compared to unirradiated embryos (solid bars) at both the 200-min $(\mathrm{P}<0.001)$ and $230-\mathrm{min}(\mathrm{P}<0.002)$ time points. Sample sizes for 180-, 200-, and 230-min samples were 66, 115, and 57, respectively, for irradiated and 44,112 , and 53 , respectively, for unirradiated embryos.

cell cycle program also led to a delay in the degradation of $b c d$ maternal message.

The development of the seven-striped transcript pattern of the segmentation gene even-skipped was independent of the nuclear density and cell cycle program

Transcription of some of the zygotic genes important for segmentation of the embryo is initiated or modulated during cycle 14. To ask whether the delay in zygotic transcription observed for $s t g$ was indicative of an overall delay in development, we examined transcription of the zygotic pattern genes even-skipped (eve). eve is a member of the pair-rule class of segmentation genes (Nüsslein-Volhard and Wieschaus 1980). Although transcription of $e v e$ can be detected as a broad domain in cycle 13, the characteristic seven-striped eve expression pattern is developed during cycle 14 (Harding et al. 1986; Macdonald et al. 1986). The initial broad expression domain is broken up into two smaller broad domains. Next, stripe 7 , the most posterior stripe, is defined, giving a two broad plus 7 pattern. The remainder of the stripes are defined by the subdivision of the two broad domains to give a series of intermediate patterns. The maturation of the seven-striped eve pattern can therefore be scored to mark the progression of the segmentation process.

The maturation of the eve expression pattern in UVirradiated embryos was examined by the same method described above for $s t g, T l$, and $b c d$. The material used for this analysis were other embryos from the same set of collections used to generate the $s t g$ results. After in situ hybridization to visualize the eve transcript, the embryos were scored for their eve expression pattern into four classes (Fig. 7a): (1) 2 Broad-1 or 2 broad domains; (2) 2 Broad $+7-2$ broad domains and a distinct stripe 7 ; (3) Intermediate-all intermediates in subdivision of the broad domains; and (4) Mature-the complete 7-striped pattern. At both the middle cycle $14(200 \pm 5 \mathrm{~min}$ AEL) and the late cycle $14(230 \pm 5$ min AEL $)$ time points, there was no difference in the distribution of eve expression patterns between the irradiated embryos and the controls. Similar results were also obtained from the analysis on DNA-injected embryos at the mid-cycle 14 time point $(\mathrm{P}=0.35, n=19$ for controls and $n=16$ for DNA-injected embryos) and the late-cycle 14 time point $(\mathrm{P}=0.44, n=26$ for controls and $n=31$ for DNA-injected embryos). The maturation of the eve expression pattern was therefore not dependent on the nuclear density or cell cycle program of the embryo.

The independence of the maturation of $e v e$ expression from the effects of nuclear density and cell cycle program was underscored by the transcript pattern in embryos that were mosaic in their cell cycle program. These were embryos that had undergone an additional local mitotic wave. The mature eve expression pattern and nuclear morphology in a control embryo are shown in Figure $7 b$. It is possible to observe both the eve expression pattern and the consistent nuclear morphology in the embryo. The eve RNA pattern and nuclear morphology in a UVirradiated embryo are shown in Figure $7 \mathrm{c}$. This embryo apparently underwent an additional mitotic wave that was initiated at the anterior pole and spread along the ventral side of the embryo. The difference in nuclear morphology resulting from the additional mitosis is clearly evidenced by the DAPI nuclear staining. In contrast to the mosaic pattern in the cell cycle program and nuclear morphology, eve expression has the mature seven-striped pattern in both halves of the embryo. The stripes are weaker and narrower in the half of the embryo that had undergone the additional division.

Transcription of the homeotic gene Antennapedia was independent of the nuclear density and cell cycle program

The homeotic gene Antennapedia (Antp) is required for thoracic development. The P2 promoter of Antp drives a three-striped pattern of expression that is initially tran- 

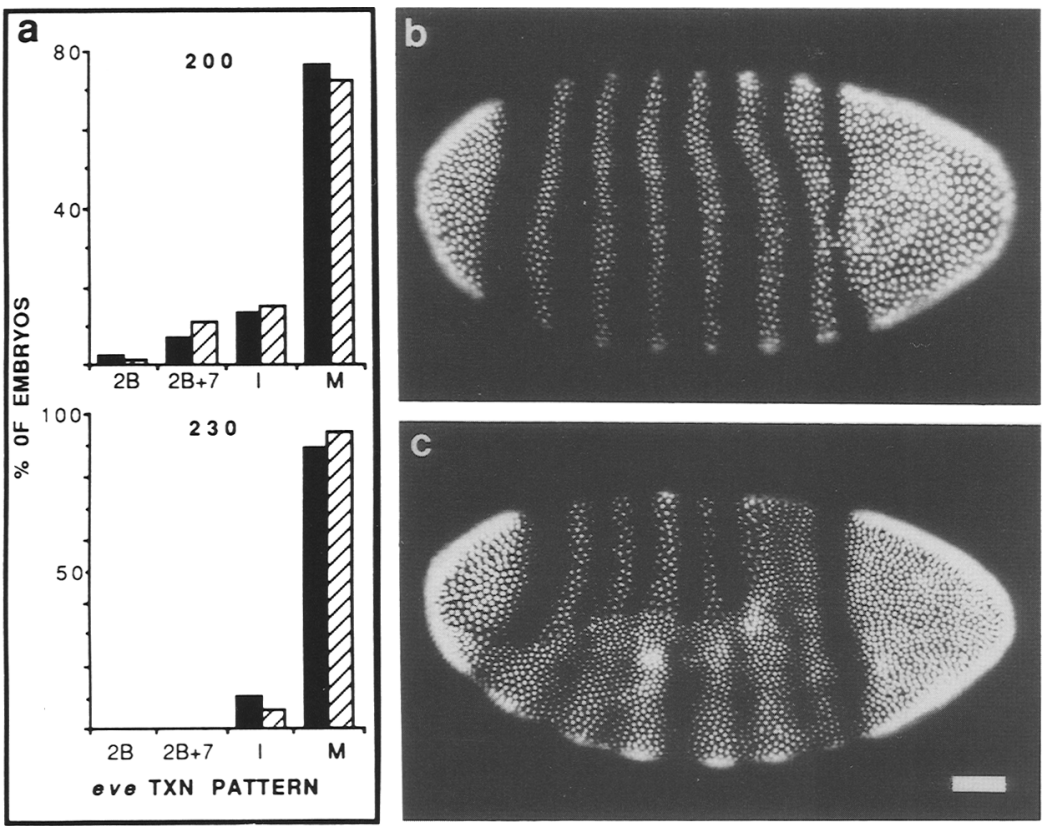

Figure 7. The expression (TXN) of the zygotic pattern gene eve was not affected in UV-irradiated embryos. The eve transcript was visualized by hybridization with a nonradioactive probe followed by detection with an antibody conjugated to alkaline phosphatase. Because of the apical accumulation of eve transcript, the product of the alkaline phosphatase reaction quenched DAPI nuclear staining in regions with high levels of eve transcript. They were then scored as belonging to one of four classes. The details of the scoring classes are described in the text. $(a)$ Summary of the observations made on the irradiated (hatched bars) and unirradiated (solid bars) embryos. Sample sizes for the 200- and 230-min time points were 65 and 36 , respectively, for irradiated and 80 and 19 , respectively, for the unirradiated embryos. At both the 200-min $(P=0.87)$ and the $230-\mathrm{min} \quad(P=0.90)$ time points the distribution of expression patterns among the irradiated embryos is not significantly different from the distribution in unirradiated embryos. (b) The mature eve expression pattern and the consistent nuclear morphology in an unirradiated control embryo. The mosaic DAPI staining pattern of the irradiated embryo in $c$ shows that it has undergone an additional local division which was initiated at the anterior pole and has extended farther ventral than dorsal. In contrast to the mosaic nuclear morphology, only the mature seven-striped eve expression pattern is observed in this embryo. Bar, $50 \mu \mathrm{m}$.

scribed in cycle 14 (Harding and Levine 1988; Irish et al. 1989; Jorgensen 1989; Bermingham et al. 1990). The striped domains are transcribed sequentially: first, a full stripe corresponding to parasegment 4 (PS4), then a partial stripe extending over only the ventral half of PS6 and, finally, a partial stripe extending over the dorsal half of PS14 (Fig. 8a).

The transcription from the P2 promoter of Antp in UV-irradiated embryos was examined. Again, other embryos from the same collections used for the stg hybridizations were used. The embryos were scored into four classes corresponding to the number of stripes of Antp expression (0, PS4, PS4 + PS6, and PS4 + PS6 + PS14). The distribution of Antp expression patterns among the UV-irradiated embryos was not different from that among the control embryos at either the middle cycle 14 $(\mathrm{P}=0.51, n=113$ for controls and $n=84$ for UV-irradiated embryos $)$ or the late cycle 14 time point $(P=0.50$, $n=145$ for controls and $n=97$ for UV-irradiated embryos). It was also possible to find embryos that were mosaic for their cell cycle program and yet not mosaic for their Antp expression pattern. Figure 8b shows the DAPI nuclear staining of an embryo that underwent an anterior 15 type of compensation, with a mitotic wave initiated at the anterior pole and spreading approximately one-third of the way across the embryo. In contrast to the mosaic pattern in nuclear morphology, the stripes of Antp transcription clearly cross the mosaic boundary (Fig. 8c). Therefore, the transcription of this homeotic gene was also independent of the nuclear density or cell cycle program of the embryo.

\section{Discussion}

In the most simplified view, the concurrent changes in the pattern of transcripts seen at cycle 14 in Drosophila or at mid-blastula transition in Xenopus could result from a single regulatory signal that is cycle dependent. Nuclear density and cell cycle program have been proposed to be important regulatory factors for the overall level of zygotic transcription (Edgar and Schubiger 1986; Kimelman et al. 1987). We have demonstrated that there is not a single, cycle-dependent switch for all gene expression in Drosophila. We examined five transcripts, which undergo changes in transcript pattern during cycle 14 , in embryos with experimentally generated alterations in nuclear density and cell cycle program. From this analysis, we propose two modes for the temporal regulation of changes in transcript pattern in the early embryo: (1) Nuclear density/cycle dependent: Both the degradation of maternal message and the initiation of zygotic transcription from $s t g$ and $T l$ were influenced by changes in nuclear density and cell cycle program. In addition, these changes also affected the degradation of maternal $b c d$ message. The degradation of maternal messages such as $s t g, T l$, and $b c d$ messages may be promoted by zygotic transcription (see below). Therefore, these processes may also serve as indicators of zygotic transcriptional activity. (2) Cycle independent or time correlated: The development of the seven-striped transcription pattern of eve and the initiation of transcription from the Ant $p$ P2 promoter was apparently independent of the nuclear density and cell cycle program of the 

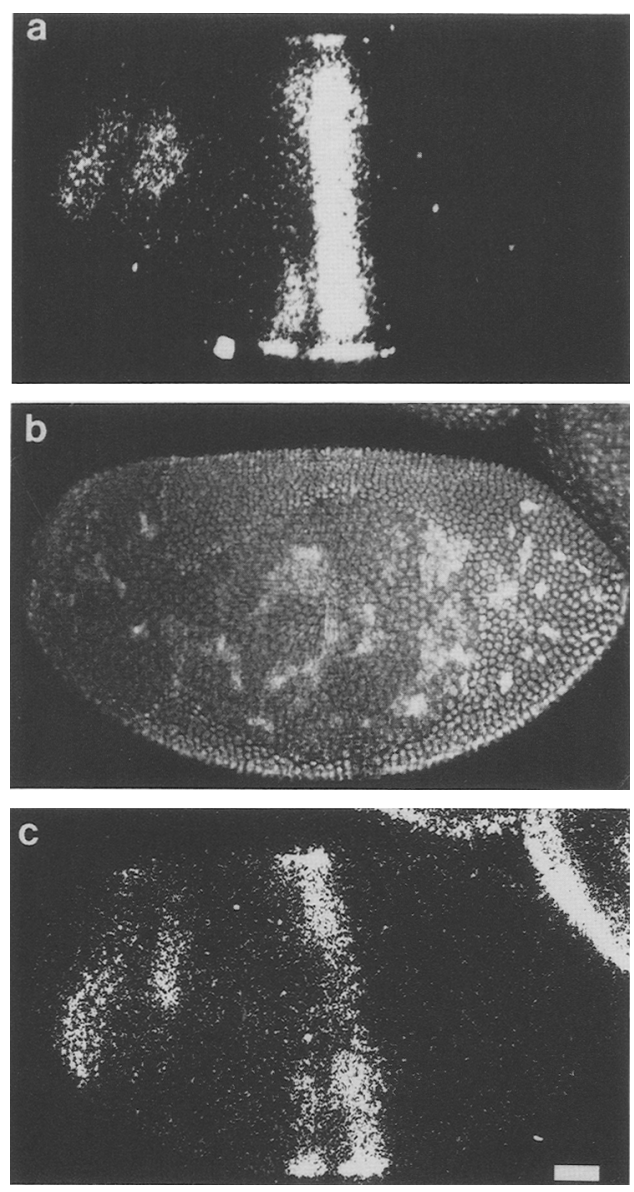

Figure 8. Transcription from the $\mathrm{P} 2$ promoter of the homeotic gene Antp was not affected in UV-irradiated embryos. UV-irradiated embryos and unirradiated control embryos were fixed in parallel, and transcription from the P2 promoter was visualized by in situ hybridization. The in situ hybridization of the unirradiated embryo in $a$ shows the control three-striped pattern of P2 transcription. Note that this pattern consists of a full circumferential stripe corresponding to PS4, a ventral-limited half stripe in PS6, and a dorsal-limited half stripe in PS14. The DAPIstained image of the irradiated embryo in $b$ shows that the embryo is mosaic in nuclear morphology as a result of the additional local mitotic wave that was initiated at the anterior pole of the embryo and extended farther ventrally. In situ hybridization of this embryo in $c$ shows that transcription of the circumferential stripe in PS4 and the ventral-limited stripe in PS6 is observed on both sides of the mosaic boundary. The PS14 stripe is present but is completely within the region of the embryo in which an extra division did not occur. $(a-c)$ An oblique dorsal view of the embryo is shown with the anterior end to the right. Bar, $50 \mu \mathrm{m}$.

embryo. Thus, this transcription may simply be dependent on developmental time.

The nucleo-cytoplasmic ratio is read and interpreted locally to regulate the cell cycle program in the embryo

The nucleo-cytoplasmic ratio has been proposed to reg- ulate the mitotic cycle in the early embryo. The ratio can be changed by increasing or decreasing the amount of DNA. This change in ratio is thought to be responsible for the increased and decreased numbers of mitotic divisions observed in haploid and polyploid embryos, respectively (Signoret and Lefresne 1971; Newport and Kirschner 1982; Edgar et al. 1986). Ligation or constriction experiments change the nucleo-cytoplasmic ratio by simultaneously changing both the number of nuclei and the amount of cytoplasm. The number of mitoses initiated in each embryo fragment is dependent on the nucleo-cytoplasmic ratio of the fragment (Kobayakawa and Kubota 1981; Newport and Kirschner 1982; Edgar et al. 1986). UV irradiation and DNA injection both altered the nucleo-cytoplasmic ratio by decreasing the number of dividing nuclei. This deficiency was corrected by the initiation of additional mitoses. Because UV irradiation and DNA injection resulted in a localized loss of nuclei in the syncytial embryo, the observed correction by local mitotic divisions indicated that the ratio was read and interpreted locally rather than globally. As the two experimental manipulations that we employed were so different, the response of the embryo seems to depend solely on the nucleo-cytoplasmic ratio in the embryo and not on the manner in which the ratio was altered.

Mitotic control in the embryo normally undergoes a transition in cycle 14 between the maternally controlled early divisions and the zygotically controlled divisions that follow cellularization. This transition is mimicked by the maternal-to-zygotic transition in expression of the gene $s t g$, which has been proposed to be a dose-dependent regulator of the zygotically controlled divisions (Edgar and O'Farrell 1989). The observed delay in the onset of the stg transition in the UV-irradiated and DNA-injected embryos indicated that the change in the nucleo-cytoplasmic ratio prolonged the maintenance of the maternal stg transcript as it induced an elongation of the maternally driven cell cycle program. The mosaic expression of $s t g$ in embryos with additional local mitotic waves indicates that the stg transition, like the cell cycle program, is controlled locally. Therefore, the observations on UV-irradiated and DNA-injected embryos suggest that the nucleo-cytoplasmic ratio controls the transition in stg expression in much the same way as it controls the cell cycle program.

\section{How might the nucleo-cytoplasmic ratio regulate changes in stg, $\mathrm{Tl}$, and bcd transcript patterns?}

The nucleo-cytoplasmic ratio might directly control the degradation of $s t g, T l$, and $b c d$ messages or indirectly control degradation through changes in the cell cycle program. O'Farrell and co-workers (1989) have proposed that degradation of maternal stg message is promoted by early transcription products and that the accumulation of these products is regulated by the cell cycle program in the embryo. This may be a general mechanism for the regulation of message stability. Consistent with this hypothesis, we have preliminary evidence that degradation of $T l$ and $b c d$ messages is promoted by zygotic transcrip- 
tion (G.K. Yasuda, unpubl.; D. Pritchard, pers. comm.). The cycle dependence of $s t g, T l$, and bcd maternal message stability might therefore reflect an underlying cycle dependence for the transcription of genes required for maternal message degradation. Although transcription of the genes required for degradation might initiate at cycle 10 , accumulation of their transcripts might then be regulated by the length of interphase. This method of transcriptional regulation was proposed by Edgar and Schubiger (1986) as a general mechanism for control of the zygotic genome.

The delay in the initiation of zygotic stg and $T 1$ transcription observed in UV-irradiated and DNA-injected embryos is not explained as easily by a simple cycledependent process. Although the entire transition was delayed, the time between degradation of maternal message and initiation of zygotic transcription did not obviously change in the experimental embryos. Therefore, the cell cycle program could also indirectly regulate $z y-$ gotic transcription of the $s t g$ and $T l$ genes simply because the initiation of transcription could require degradation of the maternal message. A possible mechanism for this type of control would be that the products of the stg and $T l$ gene negatively regulate their own transcription in some way. Possible support for such a mechanism comes from the observation that heterozygosity for stg has no apparent effect on cycle timing even though stg is believed to regulate the cycle in a dose-dependent manner. However, the feedback regulation cannot be direct because the proteins encoded by the stg and $T l$ genes are not predicted to be transcription factors (Hashimoto et al. 1988; Edgar and O'Farrell 1989). An alternative hypothesis is that the initiation of zygotic stg and $T 1$ transcription is cycle regulated by a similar mechanism as the degradation of maternal messages. That is, zygotic stg and $\mathrm{Tl}$ transcription may also be promoted by earlier transcription products whose accumulation is cycle dependent. The parallel delay in maternal message degradation and zygotic transcription that was observed in the irradiated embryos could therefore result from the cycle dependent accumulation of two different sets of products.

\section{The zygotic pattern genes eve and Antp may be members of a large class of zygotically expressed genes that are regulated in response to developmental time}

The embryo also undergoes a maternal-to-zygotic transition in the process of pattern formation. In contrast to our findings for the transition in mitotic control, changes in the nucleo-cytoplasmic ratio did not apparently affect the expression of the zygotic pattern genes. We found that maturation of the seven-striped eve transcription pattern and patterned initiation of Antp transcription in UV-irradiated embryos occurred at the same time as in control embryos. eve and Antp transcripts are spatially regulated by the gap and pair-rule classes of genes. Both positive and negative regulation is required for the proper temporal and spatial expression of these transcripts (Carroll and Vavra 1989; Edgar et al. 1989; Goto et al. 1989; Harding et al. 1989; Irish et al. 1989; Jorgensen 1989; Bermingham et al. 1990). Because the products of the gap and pair-rule genes are required for the cycle-independent changes in eve and Antp transcript pattern, it may be possible to generalize the results obtained for eve and Antp to other segmentation genes.

The obvious interpretation of our results is that these genes are regulated in some way by developmental time. To explain the behavior of these genes, we propose a modification of the previous hypothesis that transcription is regulated by the length of interphase time (Edgar and Schubiger 1986). In this earlier study, nuclear migration at cycle 10 in the embryo was defined as the time when the entire genome becomes transcriptionally "competent." Rather, our results are consistent with selective regulation of the time when individual genes or groups of genes acquire competence. Once a gene is competent for transcription, the amount of message transcribed from that gene then depends on the length of time the nucleus is in interphase. The dependence of transcript level on interphase time may explain the narrower stripes of eve transcript in the part of the embryo that had undergone an extra division in Figure 6c.

The apparent contradiction between the results presented in this study and those reported by Edgar and Schubiger (1986) derives from the basic difference between the two sets of experiments. In the earlier study overall transcription was examined by injecting radioactive precursors into the embryo. However, these investigators were only able to look specifically at the transcription of a limited number of RNA species because they had to rely on message size to distinguish between transcripts. Any differences in behavior of subsets of genes encoding longer messages, such as the ones examined in this study, would be lost in the signal from transcription of other genes encoding messages of similar size. In addition, transcriptional competence was defined by experiments in which translation was repressed and the nuclei were locked in an interphase state for $60 \mathrm{~min}$ or longer. This led to the conclusion that cycle 10 nuclei were able to transcribe in the absence of new translation products if given $60 \mathrm{~min}$ in interphase. However, the experiment did not address whether different subsets of genes were transcribed at different times during this 60 min interphase. Therefore, these experiments did not examine the possibility that different genes might acquire competence at different interphase times.

If a temporal regulation system based on developmental time exists, what developmental event turns on the counting mechanism? Evidence that this event is nuclear migration comes from a reconsideration of the data from earlier studies on the interaction between transcription and the cell cycle. Edgar et al. (1986) compared transcription initiation and cell cycle program in haploid embryos to that in diploid embryos by monitoring the initiation of transcription of the large ribosomal precursor RNA (rpRNA). They noted that there was not a perfect correlation between transcription and interphase time because a 16-min cycle 13 in the haploid embryos 
was sufficient for rpRNA transcription, yet a 16-min cycle 12 in the diploid embryos was not sufficient. This lack of expression was not an effect of nucleo-cytoplasmic ratio because a cycle 13 haploid embryo has about the same ratio as a cycle 12 diploid embryo. However, transcription of rpRNA is initiated $\sim 50$ min after nuclear migration in both the haploids and diploids. In keeping with this proposed explanation, gastrulation movements are also initiated at the same time after migration in the haploid and diploid embryos. Further evidence for the triggering of the timing mechanism at the time of nuclear migration comes from observations made on transcription in embryos from mothers homozygous for the mutation gs(1)N26. This maternal-effect mutation causes a delayed migration of nuclei to the periphery of the posterior pole (Niki and Okada 1981). In these embryos, the expression of the pair-rule gene fushi tarazu is also delayed at the posterior pole ( $\mathrm{L}$. Boring, pers. comm.). These observations are therefore consistent with the proposal that the counting of developmental time is initiated at the time of nuclear migration for the rpRNA and fushi tarazu genes.

\section{Multiple regulatory mechanisms generate concerted developmental events}

There are two basic ways to coordinate the regulation of separate developmental events. One is to have the events coordinately controlled either directly or indirectly by a single parameter. The regulation of all the events of the mitotic cycle by the level of cyclin protein is an example of such single parameter control (Murray and Kirschner 1989). The second way is to have multiple regulatory mechanisms that act in parallel to produce coordinate developmental events. In Schizosaccharomyces pombe, there is evidence that the cyclical increase in the enzyme nucleotide diphosphate kinase and in the production of $\mathrm{CO}_{2}$ is regulated independently of the cell cycle /Creanor and Mitchison 1986; Novak and Mitchison 1986). In Drosophila, the zygotic expression of $s t g, T l$, eve, and Antp is temporally coordinated in normal development. This synchrony, however, results from the regulation of stg and $T l$ by the nucleo-cytoplasmic ratio and of $e v e$ and Antp by some other mechanism that may depend on developmental time.

In higher organisms, studies of the influence of developmental time on regulation have involved predominantly descriptions of the morphogenetic movements (Cooke and Smith 1990). In the cases where timing of molecular events was investigated, the embryos were generally arrested in their morphogenetic movements making it impossible to directly compare the temporal regulation of molecular and morphogenetic events in a developing system. Our observations provide a clear example of the independent temporal regulation of these events in the developing organism.

It has become increasingly clear that the single major developmental transition observed in a number of organisms may mask the existence of multiple, separate transitions that are normally regulated in parallel with one another. In Drosophila, the major developmental transition in cycle 14-activation of the zygotic genome and transition in the cell cycle program-can be subdivided into "minor" transitions that are regulated by different timing mechanisms. By altering a single parameter in the Drosophila embryo, we have shown that transcriptional activation alone requires at least two regulatory mechanisms that are responsible for the activation of subsets of the zygotic genome. Therefore, it would not be surprising to find that the underlying network for the temporal regulation of the events in embryonic development rivals the complexity of the network for spatial patterning of the embryo.

\section{Materials and methods}

Stocks, egg collections, and staging

A Sevelen wild-type stock was used for the majority of the observations. Eggs from 3- to 7-day-old female flies were precollected for $1 \mathrm{hr}$ on fresh media and then for 30,15, and $15 \mathrm{~min}$ on $5 \%$ agar plates supplemented with acetic acid and yeast. All precollections were done at $25^{\circ} \mathrm{C}$. Following the precollections, eggs were collected for $10 \mathrm{~min}$ at $25^{\circ} \mathrm{C}$. Subsequent development was at $21-23^{\circ} \mathrm{C}$.

\section{UV irradiation}

Irradiations were done with the TM36 Transilluminator from UVP, Inc. This unit produces a maximal output at $\sim 320 \mathrm{~nm}$. Irradiation was done at a dose rate of $13.5 \mathrm{~J} / \mathrm{m}^{2}$ per sec (determined with a Ys1-Kettering model 65 Radiometer) for $12-13 \mathrm{sec}$ for a total dose of $\sim 170 \mathrm{~J} / \mathrm{m}^{2}$. Embryos were hand-dechorionated on double-stick tape and arranged on parafilm to expose primarily the anterior tip of the embryo to UV irradiation. Irradiation was done at $75 \pm 5 \mathrm{~min}$ AEL (equivalent to cycle 6 or 7 ), and fixation times are noted in the text.

\section{DNA injection}

The injections were performed with a Leitz micromanipulator and a Wild compound microscope, according to Foe and Alberts (1983). Embryos were hand-dechorionated on double-stick tape and lined up on a coverslip on a strip of double-stick tape glue dissolved with heptane. Drawn-out 50- $\mu$ l Drummond capillary pipettes were used as injection needles. Similar effects on nuclear loss and cell cycle program were observed with DNA from a variety of different sources (J. Baker, C. Kiehle, and G. Schubiger, unpubl.). In the experiments reported here, the DNA was a pBR322 plasmid containing the leu2 gene and regions of the $2 \mu$ plasmid from Saccharomyces cerevisiae, as well as the Drosophila Gart locus (provided by S. Henikoff). The DNA was injected at a concentration of $50 \mu \mathrm{g} / \mathrm{ml}$ in injection buffer (Foe and Alberts 1983) at $45 \pm 5$ min AEL (cycle 3 or 4 ).

\section{Developmental timing}

Developmental timing of cycles 9-15 was followed by direct observation or by time-lapse video microscopy of developing embryos. Direct observations of a few embryos at a time were made by using phase-contrast optics. Observations were made every $2-3 \mathrm{~min}$ at $22^{\circ} \mathrm{C}$. Time-lapse video microscopy was done according to Foe and Alberts (1983) with differential interference contrast (DIC) optics and the following components: a Mti 65 video camera, a Gyyr time-lapse video recorder, and a Koyo black/white monitor. 


\section{In situ hybridization}

UV-irradiated and DNA-injected embryos were fixed in a bilayer of heptane and $4 \%$ formaldehyde in PBS (130 mM NaCl, 10 $\mathrm{mM} \mathrm{NaPO}_{4}$ at $\mathrm{pH}$ 7.0). UV-irradiated embryos were then devitellinized in a bilayer of heptane and methanol. DNA-injected embryos were hand devitellinized with tungsten needles. All embryos were then stored in ethanol at $-20^{\circ} \mathrm{C}$. Hybridizations were to whole embryos by using either ${ }^{35} \mathrm{~S}$-labeled RNA probes, as described in Edgar and O'Farrell (1989), or digoxygenin-labeled DNA probes, as described in Tautz and Pfeifle (1989) and modified by Rick Garber (pers. comm.). After scoring the embryos into classes, as described in the text, the control and experimental embryos were compared by maximum-likelihood estimation of the equality of two multinomial distributions.

\section{Acknowledgments}

We acknowledge Caroline Kiehle and Cindy Sherbert for their assistance in the initial DNA-injection and UV-irradiation studies. The probe constructs were kindly provided by Bruce Edgar, Richard Garber, Michael Levine, and David Schneider. We thank Landin Boring, Richard Garber, and David Pritchard for communicating results prior to publication. We also thank David Kimelman, Andrew Murray, Barbara Wakimoto, and Lauren Yasuda for their critical comments on this manuscript. This work was supported by a predoctoral fellowship from the $\mathrm{Na}$ tional Science Foundation to G.K.Y. and a research grant (GM33656) from the National Institutes of Health to G.S.

The publication costs of this article were defrayed in part by payment of page charges. This article must therefore be hereby marked "advertisement" in accordance with 18 USC section 1734 solely to indicate this fact.

\section{References}

Anderson, K.V. and J.A. Lengyel. 1979. Rates of synthesis of major classes of RNA in Drosophila embryos. Dev. Biol. 70: 217-231.

Anderson, K.V., G. Jürgens, and C. Nüsslein-Volhard. 1985. Establishment of dorsal-ventral polarity in the Drosophila embryo: Genetic studies on the role of the Toll gene product. Cell 42: 779-789.

Berleth, T., M. Burri, G. Thoma, D. Bopp, S. Richstein, G. Frigerio, M. Noll, and C. Nüsslein-Volhard. 1988. The role of localization of bicoid RNA in organizing the anterior pattern of the Drosophila embryo. EMBO I. 7: 1749-1756.

Bermingham, J.R., Jr., A. Martinez-Arias, M.G. Petit, and M.P. Scott. 1990. Different patterns of transcription from the two Antennapedia promoters during Drosophila embryogenesis. Development 105: 553-556.

Carroll, S.B. and S.H. Vavra. 1989. The zygotic control of Drosophila pair-rule gene expression. II. Spatial repression by gap and pair-rule gene products. Development 107: 673-683.

Chulitskaia, E.V. 1970. Desynchronization of cell divisions in the course of egg cleavage and an attempt at experimental shift of its onset. I. Embryol. Exp. Morphol. 23:359-374.

Cooke, J. and J.C. Smith. 1990. Measurement of developmental time by cells of early embryos. Cell 60: 891-894.

Creanor, J., and J.M. Mitchison. 1986. Nucleoside diphosphokinase, an enzyme with step changes in activity during the cell cycle of the fission yeast Schizosaccharomyces pombe. I. Persistence of steps after a block to the DNA-division cycle. I. Cell Sci. 86: 207-215.

Edgar, B.A. and P.H. O'Farrell. 1989. Genetic control of cell division patterns in the Drosophila embryo. Cell 57: 177187.
1990. The three postblastoderm cell cycles of Drosophila embryogenesis are regulated in G2 by string. Cell 62: 469-480.

Edgar, B.A. and G. Schubiger. 1986. Parameters controlling transcriptional activation during early Drosophila development. Cell 44: 871-877.

Edgar, B.A., C.P. Kiehle, and G. Schubiger. 1986. Cell cycle control by the nucleocytoplasmic ratio in early Drosophila development. Cell 44: 365-372.

Edgar, B.A., G.M. Odell, and G. Schubiger. 1989. A genetic switch, based on negative regulation, sharpens stripes in Drosophila embryos. Dev. Genet. 10: 124-142.

Foe, V.E. 1989. Mitotic domains reveal early commitment of cell in Drosophila embryos. Development 107: 1-22.

Foe, V.E. and B.M. Alberts. 1983. Studies of nuclear and cytoplasmic behavior during the five mitotic cycles that precede gastrulation in Drosophila embryogenesis. I. Cell Sci. 61: 31-70.

Frigerio, G., M. Burri, D. Bopp, S. Baumgartner, and M. Noll. 1986. Structure of the segmentation gene paired and the Drosophila PRD gene set as part of a gene network. Cell 47:735-746.

Gerttula, S., Y. Jin, and K.V. Anderson. 1988. Zygotic expression and activity of the Drosophila Toll gene, a gene required for embryonic dorsal-ventral pattern formation. Genetics 119: 123-133.

Goto, T., P. MacDonald, and T. Maniatis. 1989. Early and late periodic patterns of even-skipped expression are controlled by distinct regulatory elements that respond to different spatial cues. Cell 57: 413-422.

Harding, K. and M. Levine. 1988. Gap genes define the limits of Antennapedia and Bithorax gene expression during early development in Drosophila. EMBO I. 7: 205-214.

Harding, K., C. Rushlow, H.J. Doyle, T. Hoey, and M. Levine. 1986. Cross-regulatory interactions among pair-rule genes in Drosophila. Science 233: 953-959.

Harding, K., T. Hoey, R. Warrior, and M. Levine. 1989. Autoregulatory and gap gene response elements of the evenskipped promoter of Drosophila. EMBO J. 8: 1205-1212.

Hashimoto, C., K.L. Hudson, and K.V. Anderson. 1988. The Toll gene of Drosophila, required for dorsal-ventral embryonic pattern formation, encodes a transmembrane protein. Cell 52: 269-279.

Irish, V.F., A. Martinez-Arias, and M. Akam. 1989. Spatial regulation of the Antennapedia and Ultrabithorax homeotic genes during Drosophila early development. EMBO $\mathrm{I}$. 8: $1527-1537$.

Jorgensen, E.M. 1989. "Function and misfunction of the two promoters of the Antennapedia gene in Drosophila melanogaster." Ph.D. thesis, University of Washington, Seattle, Washington.

Kimelman, D., M. Kirschner, and T. Scherson. 1987. The events of the midblastula transition in Xenopus are regulated by changes in the cell cycle. Cell 48: 399-407.

Knipple, D.C., E. Seifert, U.B. Rosenberg, A. Preiss, and H. Jäckle. 1985. Spatial and temporal patterns of Krüppel gene expression in early Drosophila embryos. Nature 317: 40-44.

Kobayakawa, Y. and H. Kubota. 1981. Temporal pattern of cleavages and the onset of gastrulation in amphibian embryos developed from eggs with reduced cytoplasm. J. Embryol. Exp. Morphol. 62: 83-94.

Lamb, M.M. and C.D. Laird. 1976. Increase in nuclear poly(A)containing RNA at syncytial blastoderm in Drosophila melanogaster embryos. Dev. Biol. 52: 31-42.

Macdonald, P.M., P. Ingham, and G. Struhl. 1986. Isolation, structure, and expression of even-skipped: A second pair- 
rule gene of Drosophila containing a homeo box. Cell 47: 721-734.

McKnight, S.L. and O.L. Miller, Jr. 1976. Ultrastructural patterns of RNA synthesis during early embryogenesis of Drosophila melanogaster. Cell 8: 305-319.

Murray, A.W. and M.W. Kirschner. 1989. Dominoes and clocks: The union of two views of the cell cycle. Science 246: 614621.

Newport, J. and M. Kirschner. 1982. A major developmental transition in early Xenopus embryos. I. Characterization and timing of cellular changes at the midblastula stage. Cell 30: 675-686.

Niki, Y. and M. Okada. 1981. Isolation and characterization of grandchildless-like mutants in Drosophila melanogaster. Roux's Arch. Dev. Biol. 190: 1-10.

Novak, B. and J.M. Mitchison. 1986. Changes in the rate of $\mathrm{CO}_{2}$ production in synchronous cultures of the fission yeast Schizosaccharomyces pombe: A periodic cell cycle event persists after the DNA-division cycle has been blocked. $/$. Cell Sci. 86: 191-206.

Nüsslein-Volhard, C. and E. Wieschaus. 1980. Mutations affecting segment number and polarity in Drosophila. Nature 287: 795-801.

O'Farrell, P.H., B.A. Edgar, D. Lakich, and C. Lehner. 1989. Directing cell division during development. Science 246: 635640.

Rothe, M., U. Nauber, and H. Jäckle. 1989. Three hormone receptor-like Drosophila genes encode an identical DNA-binding finger. $E M B O$ /. 8: 3087-3094.

Signoret, J. and J. Lefresne. 1971. Contribution of l'etude de la segmentation de l'oef d'axolotl. I. Definition de la transition blastuleene. Ann. Embryol. Morphog. 4: 113-123.

Simcox, A.A. and J.H. Sang. 1983. When does determination occur in Drosophila embryos. Dev. Biol. 97: 212-221.

Tautz, D. and C. Pfeifle. 1989. A non-radioactive in situ hybridization method for the localization of specific RNAs in Drosophila embryos reveals translational control of the segmentation gene hunchback. Chromosoma 98: 81-85.

Tautz, D., R. Lehmann, H. Schnürch, R. Schuh, E. Seifert, A. Kienlin, K. Jones, and H. Jäckle. 1987. Finger protein of novel structure encoded by hunchback, a second member of the gap class of Drosophila segmentation genes. Nature 327: 383-389.

Togashi, S. and M. Okada. 1984. Arrest of intravitelline mitoses in Drosophila embryos by u.v. irradiation of the egg surface. I. Embryol. Exp. Morphol. 80: 43-61.

Yasuda, G.K., J. Baker, and G. Schubiger. 1991. Independent roles of centrosomes and DNA in organizing the Drosophila cytoskeleton. Development 111: 379-391.

Zalokar, M. and I. Erk. 1976. Division and migration of nuclei during early embryogenesis of Drosophila melanogaster. I. Microsc. Biol. Cell. 25: 97-106. 


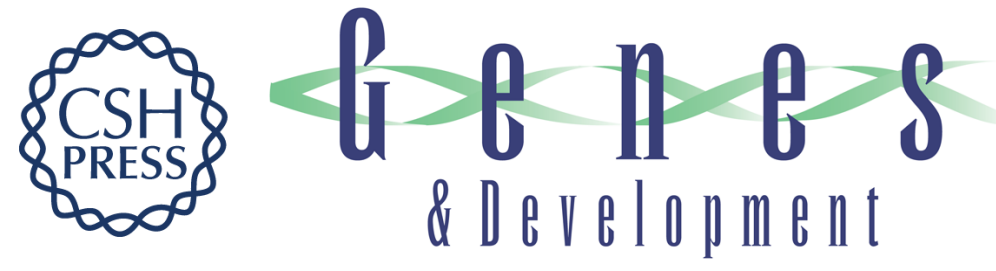

\section{Temporal regulation of gene expression in the blastoderm Drosophila embryo.}

G K Yasuda, J Baker and G Schubiger

Genes Dev. 1991, 5:

Access the most recent version at doi:10.1101/gad.5.10.1800

References This article cites 43 articles, 10 of which can be accessed free at:

http://genesdev.cshlp.org/content/5/10/1800.full.html\#ref-list-1

License

Email Alerting

Service

Receive free email alerts when new articles cite this article - sign up in the box at the top right corner of the article or click here.

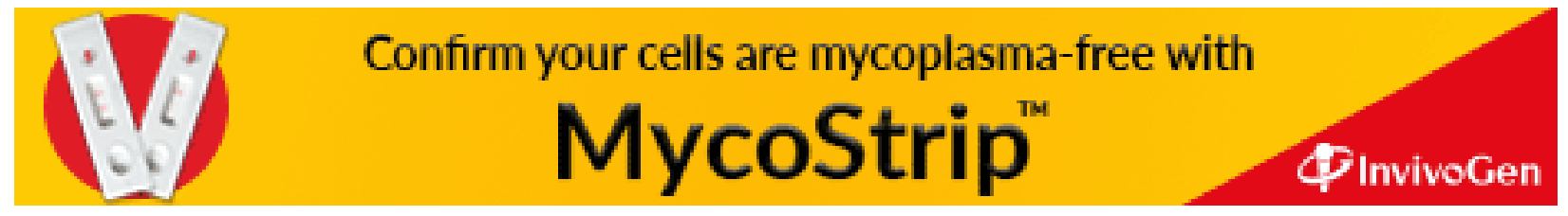

